\title{
Centrifugal Compressor
}

\section{of a Prospective Gas Compressor Unit: \\ Selection of Parameters and Sample Project Compressor}

\author{
Boris V. Bakaeva, \\ Yuri N. Pisareva, Anna A. Lysyakova ${ }^{a}$, \\ Michail M. Lentsmana ${ }^{a}$, Yuri B. Galerkin ${ }^{b}$, \\ Aleksey F. Rekstin ${ }^{\mathrm{b}}$ and Aleksandr A. Drozdov*b \\ a JSC «Iskra-Avigaz» \\ 93 Komsomolski, Perm, 614990, Russia \\ ${ }^{b}$ Peter the Great St. Petersburg Polytechnic University \\ ONTI, R\&D Laboratory "Gas dynamics of turbo machines" \\ 29 Polytechnical Str., St. Petersburg, 195251, Russia
}

Received 04.06.2017, received in revised form 16.08.2017, accepted 10.10.2017

Presented is the analysis of the optimal rpm and number of compressor stages. In the framework of the project "The creation of modern hi-tech production of design, production and installations tests, has compressing gaseous products for effective use in transport systems and technologies" grant the Ministry of education and science of the Russian Federation on the basis of RF Government decree No 218 a laboratory "Gas dynamics of turbo machines" JIST SPbPU has developed a methodology and computer software intended for advanced centrifugal compressors design. The program complex was used for geometry optimization of "Iskra-Avigaz" centrifugal compressors, and also for design of the $25 \mathrm{MW}$ compressor. Taking into account the existing trends in the development of compressor and modern experience, "Iskra-Avigaz" developed the design of the compressor and made the characteristics binding to the specified parameters in the $2^{\text {nd }}-4^{\text {th }}$ lines the North European pipeline. The high efficiency of application of the prospective compressor is shown.

Keywords: centrifugal compressor, 3D impeller, loading factor, flow rate coefficient.

Citation: Bakaev B.V., Pisarev Yu.N., Lysyakova A.A., Lentsman M.M., Galerkin Yu.B., Rekstin A.F., Drozdov A.A. Centrifugal compressor of a prospective gas compressor unit: selection of parameters and sample project compressor, J. Sib. Fed. Univ. Eng. technol., 2017, 10(8), 1062-1078. DOI: 10.17516/1999-494X-2017-10-8-1062-1078.

(C) Siberian Federal University. All rights reserved

* Corresponding author E-mail address: A_drozdi@mail.ru 


\title{
Центробежные компрессоры перспективных ГПА: выбор параметров и пример проекта компрессора
}

\author{
Б.В. Бакаев ${ }^{a}$, Ю.Н. Писарев ${ }^{a}$, \\ А.А. Лысякова ${ }^{a}$, М.М. Ленцман \\ Ю.Б. Галеркин ${ }^{\sigma}$, А.Ф. Рекстин

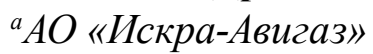 \\ Россия, 614990, Пермь, Комсомольский пр., 93 \\ ${ }^{6}$ Санкт-Петербургский политехнический университет Петра Великого, \\ Объединенный научно-технологический институт \\ Россия, 195251, Санкт-Петербург, Политехническая, 29
}

Проведен анализ оптимального числа оборотов и количества ступеней компрессоров перспективных ГПА. В рамках выполнения проекта «Создание современного высокотехнологического производства по проектированию, изготовлению и испытаниям установок, компримирующих газообразные продукты для эффективного использования в транспортных системах и технологиях» - субсидия Министерства образования и науки Российской Федерации на основании Постановления Правительства РФ № 218 лаборатория «Газовая динамика турбомашин» ОНТИ СПбПУ разработала методику и комплекс компьютерных программ, предназначенных для проектирования перспективных чентробежных компрессоров. Комплекс применен для оптимизаџии геометрии ряда проточных частей центробежных компрессоров АО «Искра-Авигаз», а также при совместной разработке геометрии проекта проточной части компрессора $25 \mathrm{MBm}$ в рамках проекта. C учетом существуюших тенденций развития компрессоростроения и современного опыта АO «Искра-Авигаз» разработала конструкиию компрессора и сделала привязку его характеристик $\kappa$ заданным параметрам 2-4-й ниток Северо-Европейского газопровода. Показана высокая эффективность применения перспективного компрессора.

Ключевые слова: иентробежный компрессор, осерадиальное рабочее колесо, коэффициент напора, коэффициент расхода.

Центробежные компрессоры применяются в различных областях промышленности, наиболее широко - при добыче и транспортировке природного газа в качестве линейных нагнетателей, дожимных компрессоров после газопроводов, когда давление газа снижается, также для закачки газа в подземные хранилища. В 2014 г. компрессорный парк ОАО «Газпром» состоял из 4254 единиц с суммарной мощностью 51000 МВт [1, 2]. Количество и мощность компрессоров газовой промышленности быстро увеличивается, поэтому при создании новых компрессоров следует принимать во внимание экономические и экологические аспекты. Обеспечение максимальной энергоэффективности очень важно. Первым и самым главным шагом в процессе создания новых компрессоров является их газодинамическое проектирование. Необходимо производить своевременную модернизацию, установкой новых сменных проточных частей (СПЧ) [3]. Компрессоры должны обеспечивать заданный массовый расход при заданном отношении давлений с максимально возможным КПД. Коэффициент полезного действия должен быть максимальным не только на расчетном, но и на нерасчетных режимах. Граница помпажа должна располагаться на максимальном удалении от расчетного режима. Это основные требования для всех турбомашин. 
Особенности газоперекачивающих агрегатов (ГПА) связаны с их параметрами проектирования. Большинство трубопроводов природного газа рассчитаны на давления 7,45...9,9 МПа. Подводные газопроводы рассчитаны на давление 12 МПа и более. Отношения давления линейных нагнетателей не превышает 1,32...1,45 [1, 2]. Безразмерное число оборотов (быстроходность) компрессора определяется его параметрами и числом оборотов ротора [4]. Величина КПД связана с быстроходностью компрессора. Линейные нагнетатели ГПА имеют две или три ступени для того, чтобы получить оптимальную быстроходность. Дожимные компрессоры и компрессоры для нагнетания газа в подземные газовые хранилища имеют отношение давления $1,7 \ldots 3,5$ и до 8 ступеней.

Компрессоры ГПА работают при малых числах Маха. Число Рейнольдса повышается с ростом давления. Характерные для компрессоров ГПА значения этих параметров способствуют достижению высокого КПД. Компрессоры с газотурбинным приводом должны затрачивать максимальную мощность на расчетном режиме работы для лучшего согласования с проводом. Проблематика статьи - количественный анализ быстроходности, информация о новой версии компьютерных программ оптимального проектирования и примеры применения, информация о перспективном трехступенчатом компрессоре 25 МВт для магистральных газопроводов с давление 9,91 МПа.

\section{Выбор оптимального числа оборотов и количества ступеней}

В течение десятилетий в отечественной газовой промышленности доминировали ГПА на конечное давление 7,45 МПа с отношением давлений 1,44 и рабочими оборотами 5200 в минуту при мощности ГТД 16 МВт. Типичный двухступенчатый компрессор с радиальными рабочими колесами (РРК) показан на рис. 1.

Газотурбинный привод накладывает ограничения на параметры компрессора. Главное из них - номинальное число оборотов, которое обычно ниже, чем нужно для выборов оптимальной проточной части компрессора. В монографиях [4] и [5] показано, что при прочих равных условиях КПД ступеней зависит от двух безразмерных газодинамических коэффициентов -

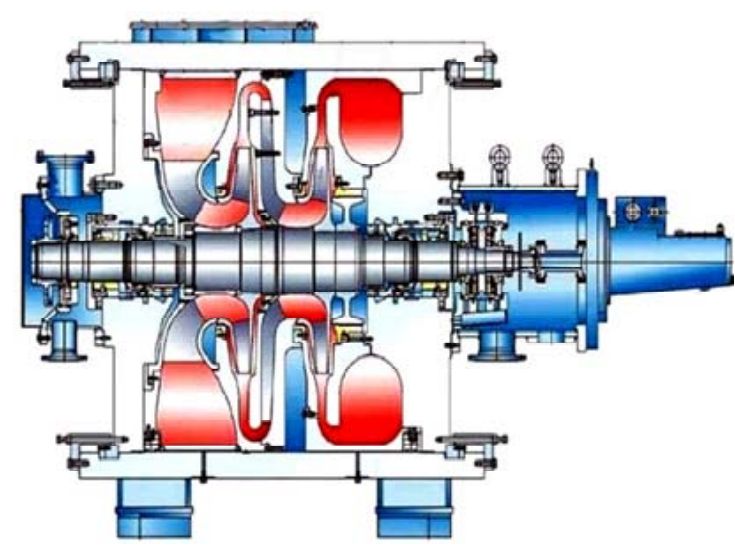

Рис. 1. Двухступенчатый компрессор линейного ГПА с радиальными рабочими колесами

Fig. 1. Two-stage pipeline compressor with 3D impellers

$$
-1064-
$$


условного коэффициента расхода $\Phi_{\text {расч }}$ и коэффициента теоретического напора $\psi_{\text {трасч }}$ на расчетном режиме:

$$
\begin{aligned}
& \Phi_{\text {pacч }}= \frac{\overline{\mathrm{m}}_{\text {расч }} / \frac{\mathrm{p}_{\mathrm{BX}}^{*}}{\mathrm{RT}_{\mathrm{Bx}}^{*}}}{\pi \frac{\mathrm{D}_{2}^{2}}{4} \mathrm{u}_{2}}(\pi=3.141), \\
& \psi_{\text {Tрасч }}=\mathrm{c}_{\mathrm{u} 2 \text { pac4 }} / \mathrm{u}_{2},
\end{aligned}
$$

где $\overline{\mathrm{m}}_{\text {расч }}$ - массовый расход на расчетном режиме, кг/с; $\mathrm{p}_{\text {вх }}^{*}$ - полное давление на выходе, Па; $\mathrm{T}_{\mathrm{Bx}}^{*}$ - полная температура на выходе, $\mathrm{K} ; \mathrm{R}$ - газовая постоянная, Дж/(кг*К); $\mathrm{u}_{2}$ - окружная скорость на выходе из рабочего колеса (РК), м/с; $\mathrm{D}_{2}$ - диаметр РК, м; $\mathrm{c}_{\text {uррасч }}$ - окружная составляющая абсолютной скорости на выходе из $\mathrm{PK,} \mathrm{м/c.}$

Эти коэффициенты связаны с расходом, напором и числом ступеней компрессора через

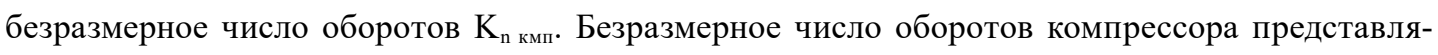
ет соотношение между массовым расходом и плотностью газа (объемным расходом), напором компрессора и числом оборотов ротора в секунду:

$$
\mathrm{K}_{\mathrm{nкмп}}=2 \sqrt{\pi} \frac{\left(\overline{\mathrm{m}} / \frac{\mathrm{p}_{\mathrm{Bx}}^{*}}{\mathrm{RT}_{\mathrm{Bx}}^{*}}\right)^{0,5}}{\mathrm{H}_{\mathrm{i}}^{0,75}} \mathrm{n},
$$

где $\mathrm{H}_{\mathrm{i}}$ - внутренний напор, Дж; $\mathrm{n}$ - число оборотов ротора, 1/c.

При приближенном анализе проблемы потерями дискового трения и протечек можно пренебречь, т.е. $\mathrm{H}_{\mathrm{i}} \approx \mathrm{H}_{\mathrm{T}}$ (где $\mathrm{H}_{\mathrm{T}}$ - теоретический напор, Дж). Теоретический напор компрессора равен сумме напоров его z ступеней: $\mathrm{H}_{\mathrm{T}}=\mathrm{h}_{\mathrm{T} 1}+\mathrm{h}_{\mathrm{T} 2}+\ldots+\mathrm{h}_{\mathrm{Tz}}$. У линейных ГПА обычно колеса имеют одинаковые диаметры и коэффициенты напора, т.е. при количестве ступеней $\mathrm{z}$ имеет место соотношение $\mathrm{h}_{\mathrm{T}}=\mathrm{H}_{\mathrm{T}} / \mathrm{z}$. В этом случае безразмерное число оборотов первой ступени связано с параметрами компрессора и числом ступеней так:

$$
\mathrm{K}_{\text {nlстрасч }}=2 \sqrt{\pi} \frac{\left(\overline{\mathrm{m}}_{\text {расч }} / \frac{\mathrm{p}_{\mathrm{Bx}}^{*}}{\mathrm{RT}_{\mathrm{Bx}}^{*}}\right)^{0,5}}{\left(\mathrm{H}_{\mathrm{T} \text { расч }} / \mathrm{z}\right)^{0,75}} \mathrm{n}(1 / \mathrm{s}) \approx \frac{\Phi_{\text {расч }}^{0,5}}{\psi_{\text {Tрасч }}^{0,75}} .
$$

Из формул $(3,4)$ следует связь между безразмерным числом оборотов компрессора и его первой ступени:

$$
\mathrm{K}_{\text {n1страсч }} \approx \mathrm{K}_{\text {пкмпт расч }} \frac{\left(\mathrm{H}_{\text {ірасч }}\right)^{0,75}}{\left(\mathrm{H}_{\text {iрасч }} / \mathrm{z}\right)^{0,75}} \approx \mathrm{K}_{\text {пкмптрасч }} \cdot \mathrm{z}^{0,75} .
$$

Из формулы (4) расчетный коэффициент расхода 1-й ступени

$$
\Phi_{1 \text { расч }}=\left(\mathrm{K}_{\text {nlстрасч }} \cdot \psi_{\text {трасч }}^{0,75}\right)^{2}=\left(\mathrm{K}_{\text {пкмптрасч }}\left(\mathrm{z} \cdot \psi_{\text {Трасч }}\right)^{0,75}\right)^{2} .
$$

В монографии [5] подробно рассмотрен вопрос о влиянии выбранных при проектировании значений $\Phi_{\text {расч}}, \psi_{\text {трасч }}$ на размеры, число оборотов и газодинамические характеристики ступени. Максимальный КПД ступеней с радиальными непространственными лопатками достижим при $\Phi_{\text {расч опт }}=0,060-0,075$. Если коэффициент расхода меньше, КПД снижается из-за роста 
трения потока о стенки узких каналов и из-за роста потерь дискового трения и протечек. Если коэффициент расхода больше, КПД снижается из-за роста кинетической энергии и потерь из-за поворота потока в широких каналах. При надлежащем проектировании пространственные лопатки осерадиальных рабочих колес лучше соответствуют сложному характеру течения и наивысший КПД может быть получен при $\Phi_{\text {расч }}$ до $0,10-0,12$. Для отдаления критического расхода от расчетной точки следует принимать значения $\psi_{\text {трасч }}=0,45-0,50$. При этих значениях $\psi_{\text {трасч }}$ КПД также может быть максимальным, а максимальная потребляемая мощность достигается на расчетном режиме или вблизи его.

С учетом изложенного оценим безразмерные параметры типичного компрессора ГПА на рис. 1. Конечное давление равно 7,45 МПа. При отношении давлений 1,44 начальное давление равно 5,174 МПа, начальная температура 288 К, на входе показатель изоэнтропы $\mathrm{k}=1,36$, газовая постоянная с учетом сжимаемости $\mathrm{zR}=453$ Дж/кг/К, среднее значение $\mathrm{k}=1,37, \mathrm{zR}=457$ Дж/кг/К. По известным термодинамическим соотношениям при КПД 0,85 и щелевых потерях 0,045 теоретический напор компрессора равен 48500 Дж/кг/К. Массовый расход компрессора 295 кг/с, объемный расход 7,44 м³/с. Безразмерное число оборотов по ф. (3) $\psi_{\text {Трасч }}=0,256$.

При $\mathrm{K}_{\mathrm{n} \text { компр }}=0,256$ и оптимальном коэффициенте напора $\psi_{\text {трасч }}=0,50$ одноступенчатый компрессор имел бы $\Phi_{\text {расч }}=0,0231$, что далеко выходит за нижний предел оптимальных значений. При двух ступенях $\mathrm{K}_{\text {nсст расч }}=0,431, \Phi_{\text {расч }}=0,0647$, при трех ступенях $\mathrm{K}_{\text {nlст расч }}=0,584, \Phi_{\text {расч }}=0,119$. То есть значения коэффициентов напора и расхода при двух ступенях вполне благоприятны для применения высокоэффективных рабочих колес с радиальными непространственными лопатками, например, представленных в [6]. При трех ступенях необходимо применить ступени на базе пространственных осерадиальных колес, о некоторых их принципах проектирования сказано ниже. Но проблема усугубляется тем, что в современных газопроводах давление повышено до 9,91 [2]. При этом объемный расход газа меньше, и, при прочих равных условиях, безразмерное число оборотов меньше (4). Это требует тщательного выбора параметров компрессора. Приводимые ниже формулы иллюстрируют вопрос количественно. Все соотношения относятся к расчетному режиму, поэтому подстрочный индекс не используется.

Потребляемая компрессором мощность $\mathrm{N}_{\text {компр }}$ равна произведению массового расхода на внутренний напор. Если пренебречь незначительной разницей между внутренним и теоретическим напором и изменением значений показателя изоэнтропы и газовой постоянной в процессе сжатия, то

$$
\mathrm{N}_{\text {компр }} \approx \overline{\mathrm{m}} \cdot \mathrm{H}_{\mathrm{T}}=\frac{\pi}{4} \cdot \Phi_{1} \cdot \mathrm{D}_{2}^{2} \cdot \mathrm{u}_{2} \frac{\mathrm{p}_{\mathrm{k}}^{*}}{\pi^{*} \cdot \mathrm{RT}_{\mathrm{Bx}}^{*}} \mathrm{H}_{\mathrm{T}} \cdot
$$

Так как $\mathrm{D}_{2}=\frac{60 \mathrm{u}_{2}}{\pi \cdot \mathrm{n}(\text { об/мин })}, \mathrm{u}_{2}=\left(\frac{\mathrm{H}_{\mathrm{T}}}{\mathrm{z} \cdot \psi_{\mathrm{T}}}\right)^{0,5}$, то оптимальное число оборотов ротора компрессора в минуту в зависимости от мощности, заданных параметров газа и выбранных параметров проектирования - коэффициентов расхода и напора - и числа ступеней предстает в виде

$$
\begin{gathered}
\mathrm{n}_{\text {(обмин) }}=\left[\Phi_{1} \cdot \frac{286,5}{\mathrm{~N}_{\text {компр }}\left(\mathrm{z} \cdot \psi_{\mathrm{T}}\right)^{1,5}} \frac{\mathrm{p}_{\mathrm{k}}^{*}}{\pi^{*} \cdot \mathrm{RT}_{\mathrm{Bx}}^{*}} \mathrm{H}_{\mathrm{T}}^{2,5}\right]^{0,5}, \\
-1066-
\end{gathered}
$$


где

$$
\mathrm{H}_{\mathrm{T}}=\frac{\mathrm{k}}{\mathrm{k}-1} \mathrm{RT}_{\mathrm{Bx}}^{*}\left(\pi^{\frac{\mathrm{k}-1}{\mathrm{k} \cdot \eta_{\mathrm{r}}^{*}}}-1\right) \text {, }
$$

где $\eta_{г}^{*}-$ гидравлический КПД.

Из формулы (8) следует, в частности, что при прочих равных условиях оптимальное число оборотов ротора обратно пропорционально корню квадратному из мощности привода. Опятьтаки при прочих равных условиях оптимальное число оборотов больше при большем конечном давлении и при всех других условиях, ведущих к снижению объемного расхода.

Современная тенденция повышения конечного давления и повышения мощности ГПА как бы идут навстречу в отношении оптимального числа оборотов. Ниже представлен анализ уравнения (8) для компрессоров мощностью 16, 25 и 32 МВт, конечным давлением 9,91 МПа, отношением давлений 1,44, начальной температурой $288 \mathrm{~K}, \mathrm{k}=1,31, \mathrm{R}=460$ Дж/кг/К, коэффициентом теоретического напора 0,5 при числе ступеней 2 и 3. Гидравлический КПД 87,5 \% (без учета щелевых потерь) принят одинаковым для всех вариантов.

Результаты расчета числа оборотов в минуту, окружной скорости в м/с и диаметра рабочих колес в метрах представлены в табл. 1-3 для компрессоров мощностью 16, 25 и 32 МВт. Обороты в минуту рассчитаны по формуле (8), окружная скорость - из величины теоретического

Таблица 1. Обороты, окружная скорость и диаметр рабочих колес компрессора 16 МВт

Table 1. Rpm, impeller speed and impellers diameter of the $16 \mathrm{MW}$ compressor

\begin{tabular}{|c|c|c|c|c|}
\hline $\mathrm{z}$ & $\Phi_{\text {1рacч }}=0,060$ & $\Phi_{\text {1рacч }}=0,085$ & $\Phi_{1 \text { pacч }}=0,10$ & $\Phi_{\text {1рacч }}=0,125$ \\
\hline 2 & $6730 / 240 / 0,680$ & $8010 / 240 / 0,570$ & $8690 / 240 / 0,530$ & $9710 / 240 / 0,470$ \\
\hline 3 & $4960 / 197 / 0,760$ & $5900197 / 0,640$ & $6400197 / 0,590$ & $7160197 / 0,530$ \\
\hline
\end{tabular}

Таблица 2. Обороты, окружная скорость и диаметр рабочих колес компрессора 25 МВт

Table 2. Rpm, impeller speed and impellers diameter of the $25 \mathrm{MW}$ compressor

\begin{tabular}{|c|c|c|c|c|}
\hline $\mathrm{z}$ & $\Phi_{\text {lpacu }}=0,060$ & $\Phi_{\text {lpacч }}=0,085$ & $\Phi_{\text {lpacu }}=0,10$ & $\Phi_{1 \text { pacu }}=0,125$ \\
\hline 2 & $5380 / 240 / 0,850$ & $6410 / 240 / 0,715$ & $6950 / 240 / 0,660$ & $7770 / 240 / 0,590$ \\
\hline 3 & $3970 / 197 / 0,950$ & $4720 / 197 / 0,800$ & $5120 / 197 / 0,730$ & $5730 / 197 / 0,660$ \\
\hline
\end{tabular}

Таблица 3. Обороты, окружная скорость и диаметр рабочих колес компрессора 32 МВт

Table 3. Rpm, impeller speed and impellers diameter of the $32 \mathrm{MW}$ compressor

\begin{tabular}{|c|c|c|c|c|}
\hline $\mathrm{z}$ & $\Phi_{\text {lpacч }}=0,060$ & $\Phi_{\text {lpacч }}=0,085$ & $\Phi_{\text {lpacч }}=0,10$ & $\Phi_{\text {lpacu }}=0,125$ \\
\hline 2 & $4760 / 240 / 0,970$ & $5660 / 240 / 0,810$ & $6140 / 240 / 0,750$ & $6870 / 240 / 0,670$ \\
\hline 3 & $3510 / 197 / 1,070$ & $4170 / 197 / 0,900$ & $4530 / 197 / 0,830$ & $5060 / 197 / 0,740$ \\
\hline
\end{tabular}


напора по формуле (9), который равен 58000 дж/кг для принятых параметров. Соотношение между оборотами, окружной скоростью и диаметром РК очевидно. В данном случае по полной формуле (8) рассчитаны обороты для компрессора 16 МВт. Для компрессоров другой мощности из (8) следует, что обороты двух компрессоров разной мощности определяет соотношение $\mathrm{n}_{2}=\mathrm{n}_{1} \sqrt{\mathrm{N}_{1} / \mathrm{N}_{2}}$.

По уравнению (8) можно проанализировать конкретный проект и подобрать наилучшее соотношение параметров проектирования ступеней - условного коэффициента напора и коэффициента теоретического напора при заданных оборотах привода. Данные табл. 1-3 показывают, что при большом давлении нагнетания и современных числах оборотов ГТД наилучшие соотношения невозможно получить при двух ступенях компрессора. Переход на трехступенчатое исполнение решает задачу. При этом получаются значения $\Phi_{\text {расч}}$, требующие применения ступеней на базе высокорасходных осерадиальных рабочих колес (OPK).

\section{Примеры применения 7-й версии комплекса программ газодинамического проектирования}

Принципы профилирования ОРК и неподвижных элементов высокорасходных ступеней изучены мало. В работах [7-10] показано, что общие принципы профилирования, изложенные в [2], применимы и к высокорасходным ступеням и представлены результаты изучения специфических проблем.

Общие принципы для ступеней со всеми типами рабочих колес таковы:

- выбор размеров входа в рабочее колесо основан на минимизации входной относительной скорости с учетом стеснения;

- высота лопаток на выходе контролирует замедление потока в рабочем колесе;

- число лопаток выбирается исходя из безразмерной нагрузки;

- входные углы лопаток обеспечивают условие безударного входа;

- выходные углы лопаток обеспечивают заданный коэффициент теоретического напоpa;

- ширина безлопаточного диффузора (БЛД) выбирается для получения угла потока, обеспечивающего безотрывное течение вплоть до границы помпажа, и т.д.

В работах [7-10] представлены результаты вычислительных экспериментов, позволяющие выбрать ряд оптимальных соотношений размеров осерадиальных рабочих колес и осерадиальных ступеней. На рис. 2 изображена структура потока в ступени до и после оптимизации размеров.

В рамках договорных обязательств перед ЗАО «Объединенные газопромышленные технологии «Искра-Авигаз» лаборатория «Газовая динамика турбомашин» ОНТИ СПбПУ разработала методику и компьютерные программы, настроенные на проектирование перспективных компрессоров с высокорасходными ступенями (7-я версия Метода универсального моделирования). Работы являются частью проекта «Создание современного высокотехнологического производства по проектированию, изготовлению и испытаниям установок, компримирующих газообразные продукты для эффективного использования в транспортных системах и технологиях» - субсидия Министерства образования и науки Российской Федерации на основании 


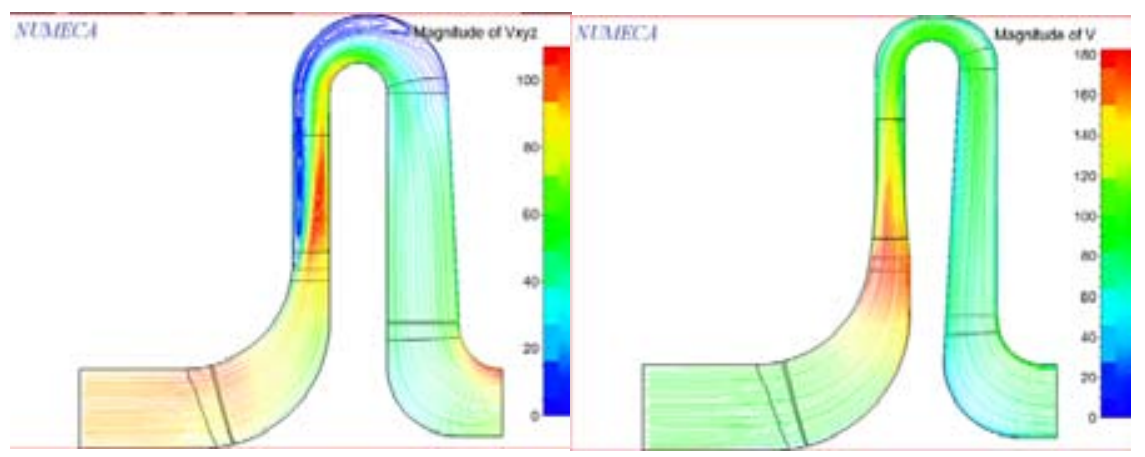

Рис. 2. Структура потока в высокорасходной ступени с ОРК до и после оптимизации размеров [7]

Fig. 2. The flow structure in high flow stage with 3D impeller before and after the dimensions optimization [7]

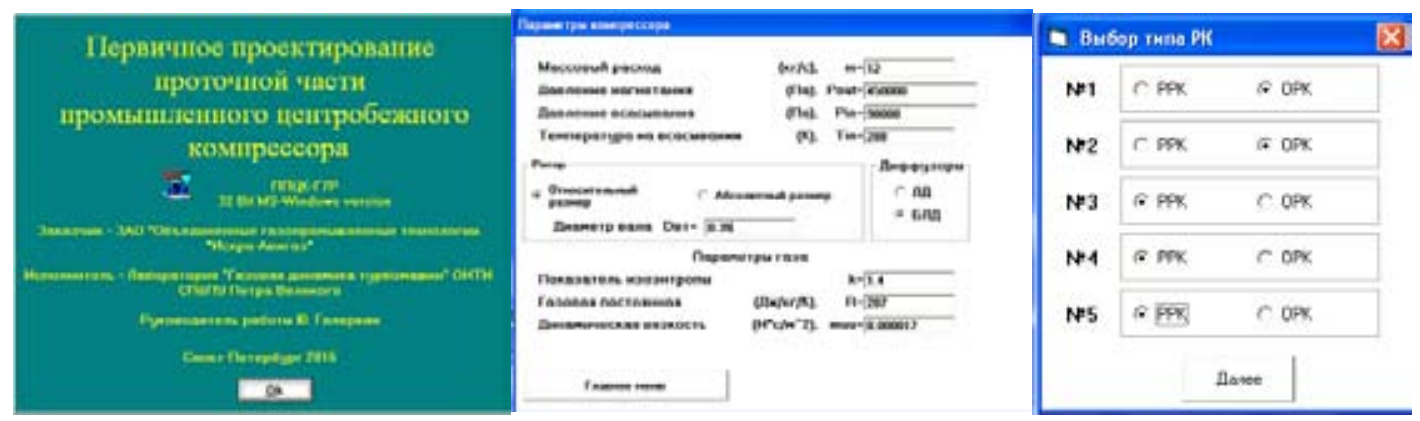

Рис. 3. Программа ППЦК-Г7Р. Заставка (слева), меню параметров компрессора (в центре), меню выбора типа РК (справа)

Fig. 3. The program NCCO-G7E. Name (left), compressor characteristic menu (center), impeller type menu (right)

Постановления Правительства РФ № 218. Комплекс программ опробован при проектировании СПЧ $16 \mathrm{MBT}$, степень сжатия 1,35 , шесть из которых успешно эксплуатируются на КС «Новоивдельская» ООО «Газпром трансгаз Югорск».

Пример вариантного расчета многоступенчатого компрессора демонстрирует универсальные возможности комплекса программ, который позволяет оптимизировать проточные части центробежных компрессоров с использованием радиальных и осерадиальных колес с разными коэффициентами напора и диаметрами. Параметры воздушного компрессора, выбранного в качестве примера, представлены в меню программы (рис. 3).

По результатам вариантного расчета выбрано пять ступеней с двумя ОРК и тремя РРК. Колеса с разными коэффициентами напора и разными диаметрами. На рис. 4 показаны основные параметры проточной части выбранного варианта. КПД рассчитан по эмпирическим уравнениям из работы [11].

Программа ППЦК-Г7Р осуществляет первичное проектирование и передачу размеров в программу для оптимизации и расчета характеристик компрессора ОПЧ-РХЦК-Г7Р. Схема проточной части по результатам предварительного проектирования изображена на рис. 5 (радиусы кривизны меридиональных обводов отличаются от действительных). 


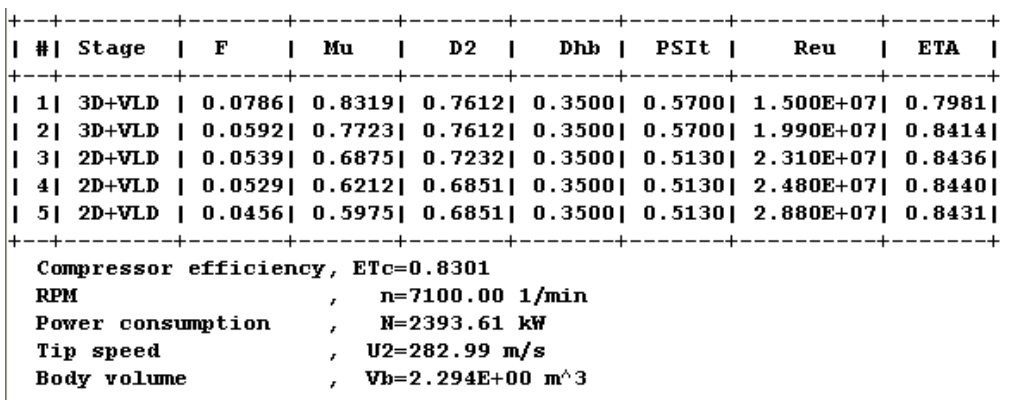

Рис. 4. Программа ППЦК-Г7Р. Основные параметры проточной части пятиступенчатого воздушного компрессора по результатам вариантного расчета

Fig. 4. The program NCCO-G7E. Main parameters a five-stage air compressor as result of variants' comparison

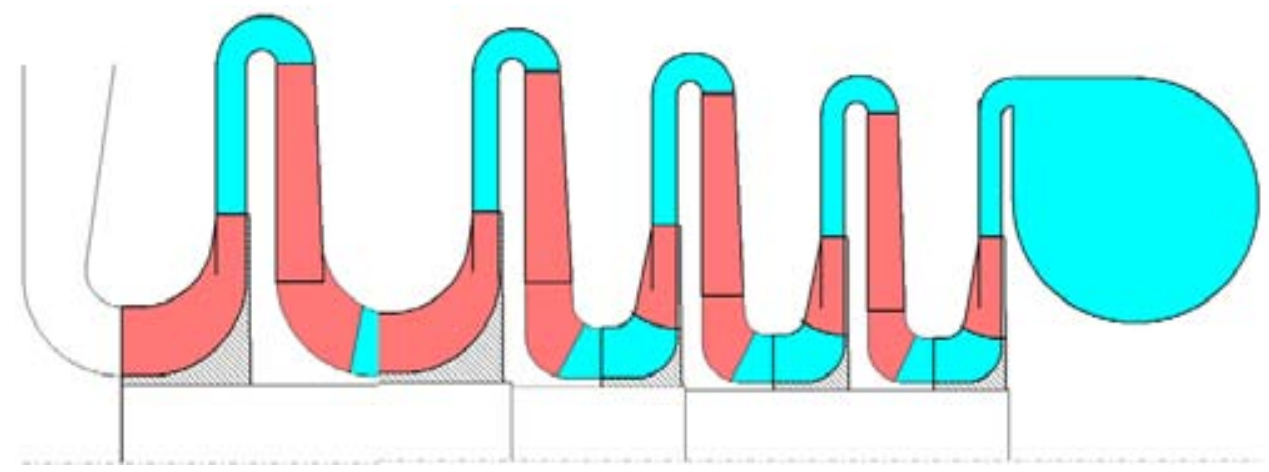

Рис. 5. Программа ОПЧ-РХЦК-Г7Р. Схема проточной части пятиступенчатого компрессора с разными типами и диаметрами рабочих колес

Fig. 5. The program CCPM-G7E. A scheme of a five-stage compressor with different types and diameters of impellers

Седьмая версия комплекса программ использована для выполнения газодинамического проектирования перспективного компрессора ЦК-25/101-1,44 для линейного ГПА трубопровода с давлением 9,91 МПа. В соответствии с анализом в предшествующем разделе число ступеней принято равным трем. На рис. 6 показано меню программы ППЦК-Г7Р с параметрами проектируемого компрессора.

В процессе вариантного расчета определяются диаметры рабочих колес, оценивается объем корпуса, рассчитываются условные коэффициенты расхода ступеней. КПД рассчитывается по эмпирическим формулам [11]. Сопоставляются варианты с разными оборотами ротора, числом ступеней и коэффициентами теоретического напора рабочих колес. В табл. 4 дан пример информации при трех ступенях и разных коэффициентах напора ОРК.

Первичное проектирование - выбор линейных размеров, отнесенных к диаметру рабочего колеса, - осуществляется по эмпирическим формулам, проверенным практикой проектирования. На рис. 7 показаны размеры ОРК в меридиональной плоскости.

Формулы оперируют линейными размерами, отнесенными к диаметру РК:

- входной диаметр рабочего колеса 


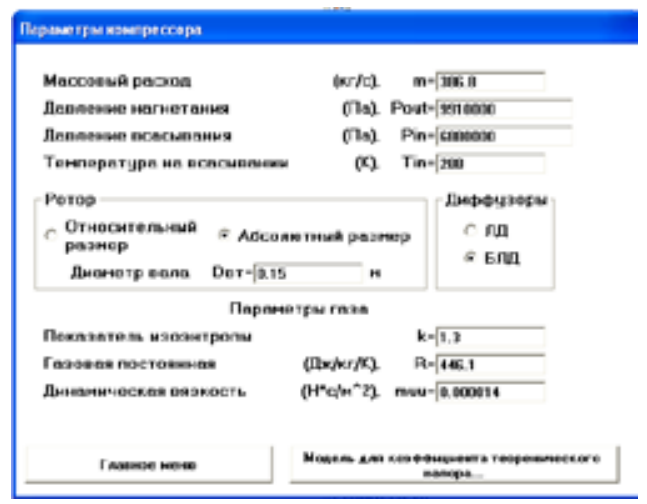

Рис. 6. Заставка программы ППЦК-Г7Р и меню ввода параметров компрессора

Fig. 6. The NCCO-G7E program name menu and input compressor parameters menu

Таблица 4. Варианты компрессора ЦК-25/101-1.44 (три ступени) при разных значениях коэффициента теоретического напора ОРК

Table 4. Variants of compressor CC-25/101-1.44 (3 stages) with different 3D impeller loading factor

***** Number of stages and Euler coefficien optimization $* * * * *$

\begin{tabular}{|c|c|c|c|c|c|c|c|c|c|c|}
\hline PSIT & ETAcomp & ETA1st & ETAlast & Nconsumpt & D2 & F1st & Flast & Vbody & U2 \\
\hline \multicolumn{10}{|c|}{ Number of stages $=3$} \\
\hline 0.3000 & 0.8709 & 0.8358 & 0.8896 & $2.180 \mathrm{E}+04$ & 0.9625 & 0.0406 & 0.0340 & $3.420 \mathrm{E}+00$ & 244.4 \\
\hline 0.3500 & 0.8730 & 0.8429 & 0.8892 & $2.180 \mathrm{E}+04$ & 0.9008 & 0.0495 & 0.0413 & $3.250 \mathrm{E}+00$ & 228.8 \\
\hline 0.4000 & 0.8742 & 0.8478 & 0.8884 & $2.180 \mathrm{E}+04$ & 0.8445 & 0.0601 & 0.0502 & $3.060 \mathrm{E}+00$ & 214.5 \\
\hline 0.4500 & 0.8750 & 0.8515 & 0.8876 & $2.180 \mathrm{E}+04$ & 0.7975 & 0.0714 & 0.0596 & $2.870 \mathrm{E}+00$ & 202.5 \\
\hline 0.5000 & 0.8754 & 0.8544 & 0.8866 & $2.180 \mathrm{E}+04$ & 0.7574 & 0.0833 & 0.0695 & $2.720 \mathrm{E}+00$ & 192.3 \\
\hline 0.5500 & 0.8750 & 0.8559 & 0.8853 & $2.180 \mathrm{E}+04$ & 0.7232 & 0.0958 & 0.0799 & $2.580 \mathrm{E}+00$ & 183.6 \\
\hline 0.6000 & 0.8737 & 0.8561 & 0.8831 & $2.180 \mathrm{E}+04$ & 0.6935 & 0.1086 & 0.0905 & $2.470 \mathrm{E}+00$ & 176.1 \\
\hline 0.6500 & 0.8714 & 0.8551 & 0.8801 & $2.190 \mathrm{E}+04$ & 0.6677 & 0.1217 & 0.1015 & $2.380 \mathrm{E}+00$ & 169.6 \\
\hline 0.7000 & 0.8681 & 0.8529 & 0.8763 & $2.200 \mathrm{E}+04$ & 0.6450 & 0.1349 & 0.1126 & $2.300 \mathrm{E}+00$ & 163.8 \\
\hline 0.7500 & 0.8639 & 0.8497 & 0.8716 & $2.210 \mathrm{E}+04$ & 0.6250 & 0.1483 & 0.1238 & $2.230 \mathrm{E}+00$ & 158.7 \\
\hline 0.8000 & 0.8587 & 0.8453 & 0.8660 & $2.220 \mathrm{E}+04$ & 0.6073 & 0.1617 & 0.1350 & $2.170 \mathrm{E}+00$ & 154.2 \\
\hline
\end{tabular}

Maximum of efficiency $=0.8754$ (Nuber of stages $=3$, PSIt $=0.50$ )

Minimum of body volume $=2.167 \mathrm{E}+00($ Nuber of stages $=3$, PSIt $=0.80)$

$$
\bar{D}_{0}=A_{D} \sqrt{\bar{D}_{\mathrm{BT}}^{2}+1,5 \Phi_{0 \text { pacu }}^{2 / 3}},
$$

где эмпирический коэффициент

$$
A_{D}=1.05-\Phi_{\text {pacu }} ;
$$

$\bar{D}_{\text {вт }}$ - относительный диаметр втулки;

- относительная высота лопаток: 


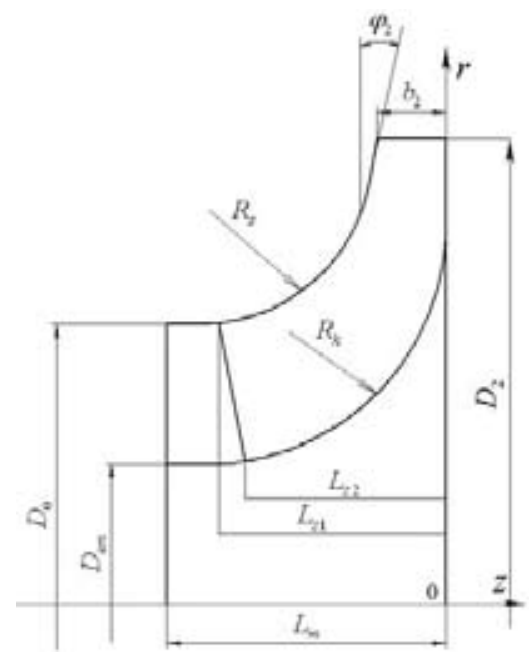

Рис. 7. Размеры осерадиального рабочего колеса в меридиональной плоскости

Fig. 7. 3D impeller dimensions in the meridional plane

$$
\bar{b}_{2}=0,9 \frac{\Phi_{0 \text { pacu }}}{1+0,6 \psi_{\text {T pacu }}} .
$$

Здесь $\Phi_{0 \text { расч }}$ - коэффициент расхода с учетом потока протечки в лабиринтном уплотнении;

- коэффициент расхода на входе в лопаточный аппарат:

$$
\varphi_{1 s}^{\prime}=\frac{\Phi_{0 \text { pacu }}}{0,765\left(\bar{D}_{0}^{2}-\bar{D}_{s m}^{2}\right)} ;
$$

- входной угол лопаток на диаметре $\bar{D}_{0}$ :

$$
\beta_{n 1 s}^{\prime}=\operatorname{arctg} \frac{\varphi_{1 s}^{\prime}}{\bar{D}_{0}}-3^{0}
$$

- выходной угол лопаток:

$$
\beta_{n 2}=12^{0}\left(1+\psi_{T \text { pacu }}\right)^{3}\left(1+\Phi_{\text {pacu }}\right) ;
$$

- осевая длина ОРК:

$$
\bar{L}_{m}=\bar{b}_{2}+0,5\left(1-\bar{D}_{0}\right)
$$

- радиус закругления покрывающего диска:

$$
\bar{R}_{s}=0,47\left(1-\bar{D}_{0}\right) ;
$$

- радиус закругления основного диска:

$$
\bar{R}_{h}=0,9 \bar{L}_{m} ;
$$

- угол наклона покрывающего диска:

$$
-1072-
$$




$$
\varphi_{2}=12^{0} ;
$$

- количество лопаток:

$$
z_{p \kappa}=2.5\left(1+3 \psi_{T \text { pacu }}\right)^{2}
$$

и т.Д.

Параметры и размеры проточной части передаются в программу ОПЧ-РХЦК-Г7Р (рис. 8).

По 7-й версии Метода универсального моделирования программа ОПЧ-РХЦК-Г7Р рассчитывает газодинамические характеристики и параметры потока в проточной части. Меридиональные очертания проточной части компрессора ЦК-25/101-1.44 после профилирования рабочих колес и неподвижных элементов показаны на рис. 9.

\section{Конструкция перспективного компрессора ЦК-25/101-1.44}

В компрессоре ЦК-25/101-1.44 использован опыт проектирования, изготовления и эксплуатации центробежных компрессоров:

- ОАО «Компрессорный комплекс» мощностью 25 МВт: 598 (4 модификации, сухие уплотнения, масляные подшипники), 588 (сухие уплотнения, масляные подшипники), 508 (сухие уплотнения, магнитные подшипники) [12-15];

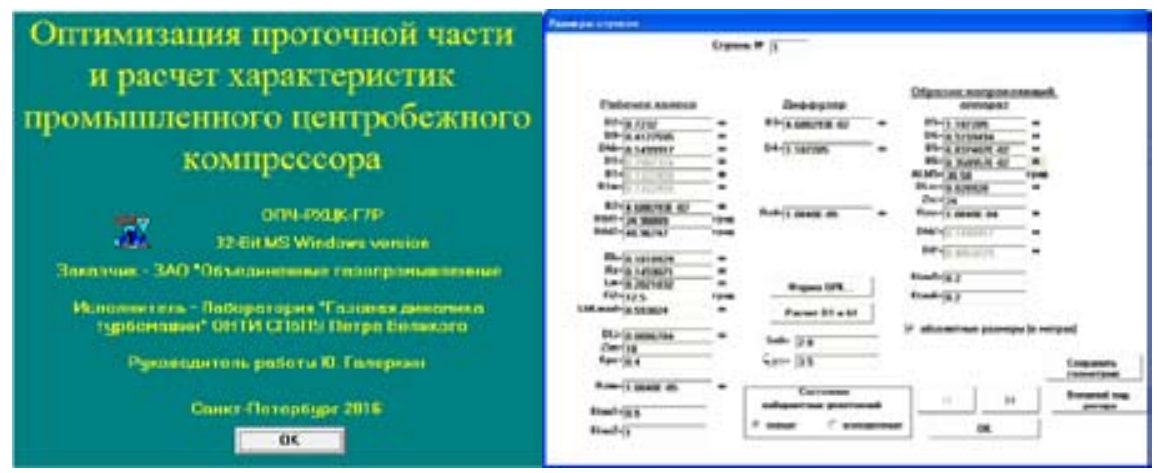

Рис. 8. Заставка программы ОПЧ-РХЦК-Г7Р и меню ввода размеров ступени

Fig. 8. The program CCPM-G7E name menu and stage dimension menu

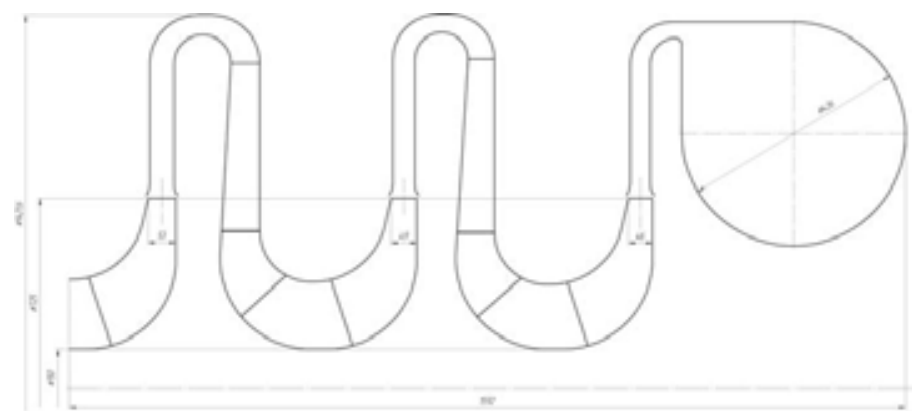

Рис. 9. Меридиональные очертания проточной части компрессора ЦК-25/101-1.44

Fig. 9. The flow path of the compressor CC-25/101-1.44 
- НПО им. Фрунзе (г. Сумы) мощностью 25 МВт: 323ГЦ2-410/70-100М (сухие уплотнения, магнитные подшипники);

- PTM-Siemens РTM 25/03-1,50/9,91; РТМ 25/04-1,60/11,9 (сухие уплотнения, масляные подшипники).

Техническое задание на газодинамический проект было сделано в соответствии с выданными исходными требованиями (Гипроспецгаз). Характеристики «степень сжатия, КПД - объемная производительность» для КС «Грязовецкая» (2-я нитка СЕГ) представлены на рис. 10. Аналогичный анализ характеристик компрессора ЦК-25/101-1.44 и эффективности его применения для ниток 3 - 4 СЕГ (КС «Грязовецкая», КС «Дивенская») показан на рис. 11, 12.

Компрессор обеспечивает гарантированную эффективность работы компрессора (КПД не ниже 87 \%) во всем диапазоне заданных параметров для различных компрессорных станций и цехов, несмотря на существенную разницу их значений.

На рис. 13 изображен продольный разрез и аксонометрический разрез компрессора.

Корпус компрессора имеет тангенциальный выходной и радиальный входной патрубки. Возможно размещение до четырех рабочих колес с увеличением степени сжатия до 1,8 . Исполнение предусматривает применение как масляных, так и магнитных подшипников. В конструкции компрессора, рекомендуемого для СЕГ, предусмотрено использование сухих уплотнений и магнитных подшипников, причем съем гильзы СГУ обеспечен без снятия роторной части магнитного подшипника.

\section{Заключение}

Анализ безразмерных параметров компрессорных ступеней указывает на необходимость тщательного выбора соотношения между мощностью компрессора, его объемной производи-

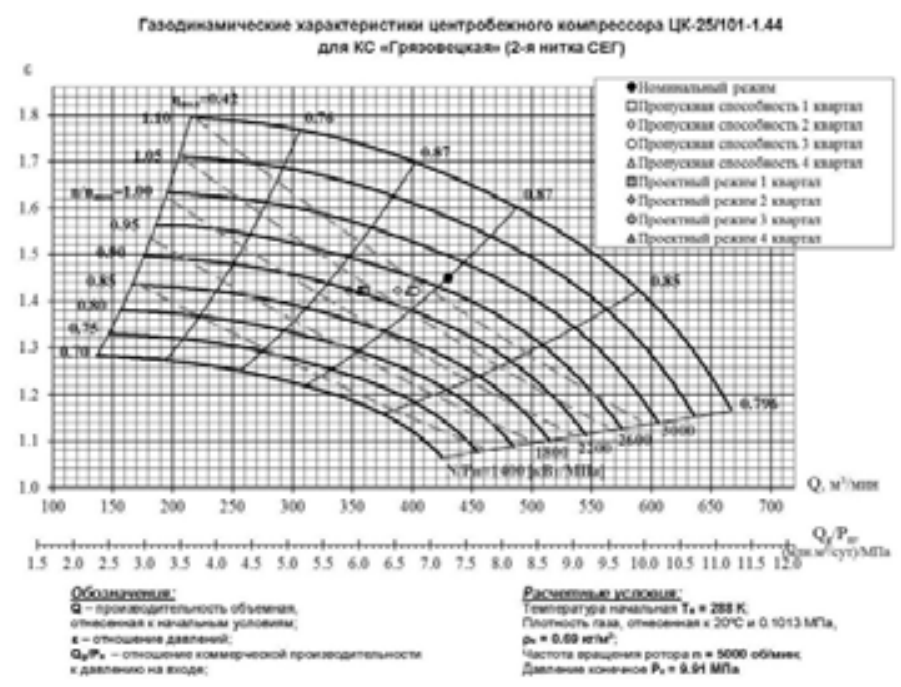

Рис. 10. Газодинамические характеристики компрессора ЦК-25/101-1.44 для КС «Грязовецкая» (2-я нитка $\mathrm{CE} \Gamma)$

Fig. 10. Gas dynamic characteristics of compressor CC-25/101-1.44. The compressor station «Gryazovetskaya» (the $2^{\text {nd }}$ line of the North European pipeline) 


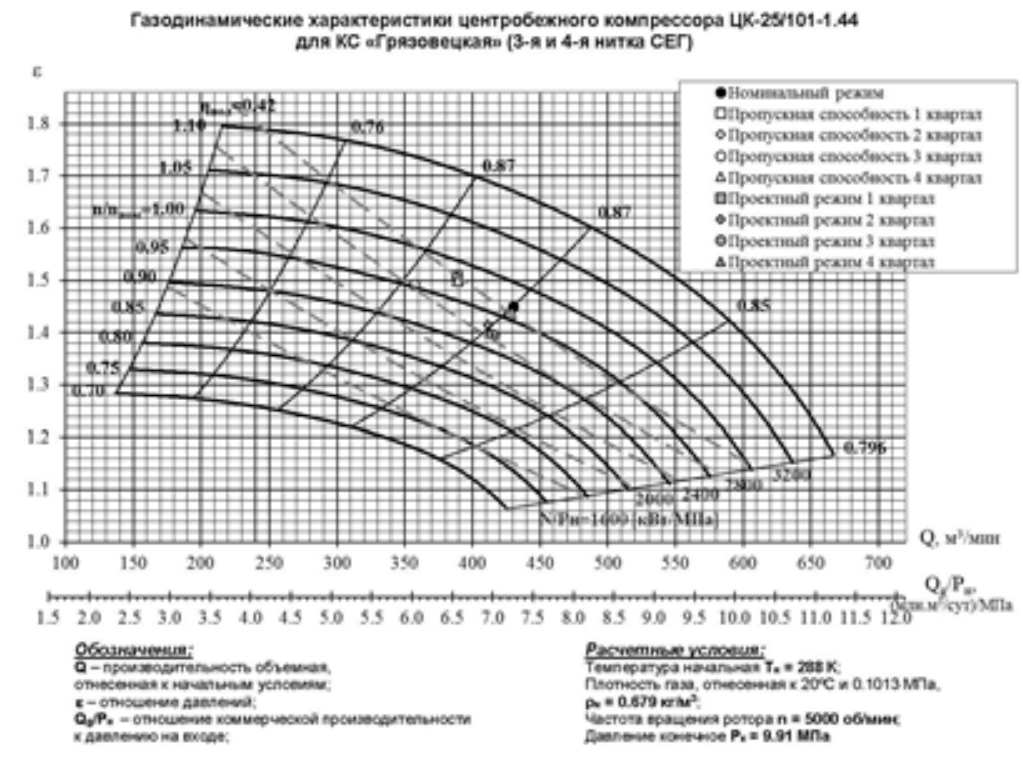

Рис. 11. Газодинамические характеристики компрессора ЦК-25/101-1.44 для КС «Грязовецкая» (3-я и 4-я нитка СЕГ)

Fig. 11. Gas dynamic characteristics of compressor CC-25/101-1.44. The compressor station «Gryazovetskaya» $\left(3^{\text {rd }}\right.$ and $4^{\text {th }}$ lines of the North European pipeline)

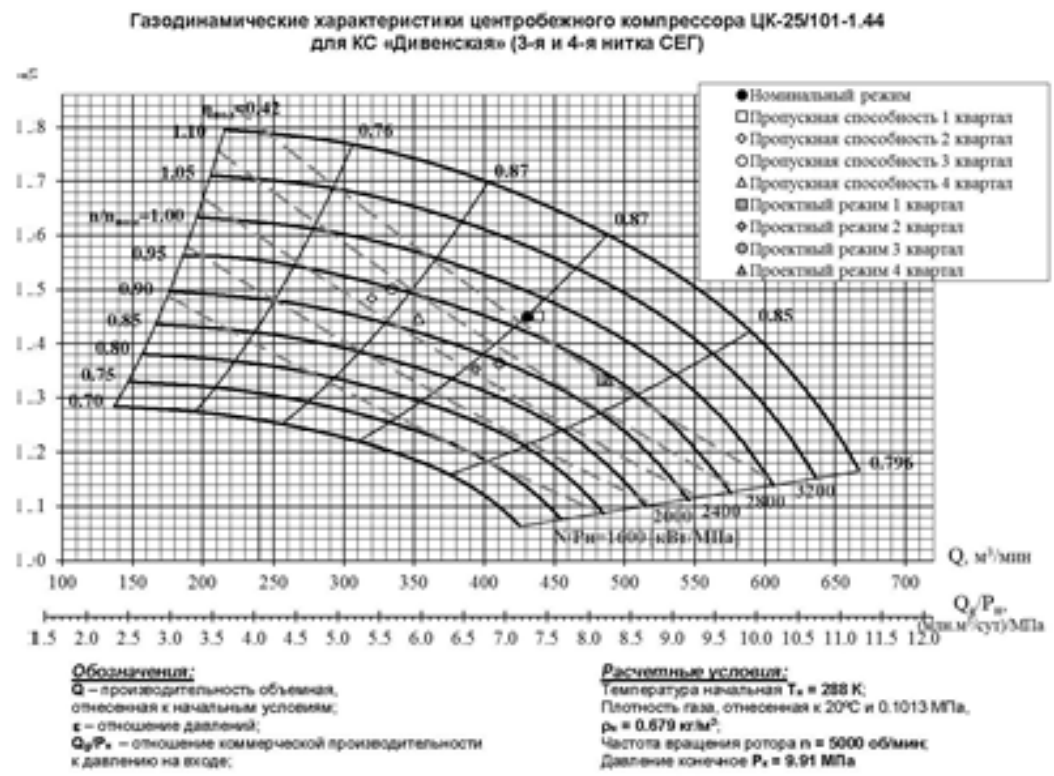

Рис. 12. Газодинамические характеристики компрессора ЦК-25/101-1.44 для КС «Дивенская» (3-я и 4-я нитки СЕГ)

Fig. 12. Gas dynamic characteristics of compressor CC-25/101-1.44. The compressor station «Diventskaya» (3 ${ }^{\text {rd }}$ and $4^{\text {th }}$ lines of the North European pipeline) 

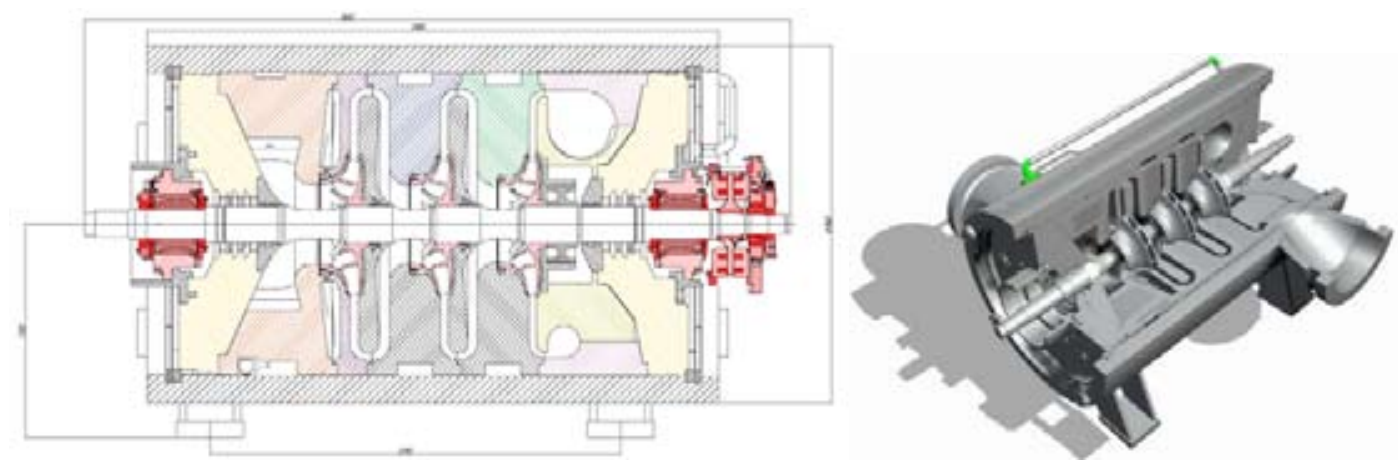

Рис. 13. Продольный разрез (слева) и аксонометрический разрез (справа) компрессора ЦК-25/101-1.44

Fig. 13. The compressor CC-25/101-1.44 views

тельностью, числом оборотов ротора и количеством ступеней. Для компрессоров линейных ГПА магистральных газопроводов с высоким давлением целесообразно применять компрессоры с тремя ступенями. Проект компрессора 25 МВт на конечное давление 9,91 МПа, выполненный АО «Искра-Авигаз» с участием НИЛ «Газовая динамика турбомашин» СПбПУ, гарантирует высокие эксплуатационные свойства и сможет стать основой для создания семейства новых компрессоров. Разработанный комплекс компьютерных программ позволяет оптимизировать выбор центробежных компрессоров и их комбинаций для сложных технологий, в составе которых применяются агрегаты различной единичной мощности, степени сжатия, расхода, состава газа, начальных и конечных условий.

\section{Благодарность}

Результаты, указанные в публикации, получены при выполнении Головным исполнителем научно-исследовательской, опытно-конструкторской технологической работы «Создание современного высокотехнологичного производства по проектированию, изготовлению и испытаниям установок, компримирующих газообразные продукты для эффективного использования в транспортных системах и технологиях». (При финансовой поддержке Правительства Российской Федерации (Минобрнауки России) - договор 02.G25.31.0140 от 01.12.2015).

\section{Список литературы}

[1] Седов В.В., Сальников С.Ю., Щуровский В.А. Современная газокомпрессорная техника - результат совместной деятельности производителей и потребителей. Компрессорная техника и пневматика, 2014, 8, 2-7. [Sedov V.V., Salnikov S.Y., Schurovski V.A. Modern gas compressor equipment - the result of joint activities of producers and consumers Compressors and Pneumatics, 2014, 8, 2-7. (in Russian)]

[2] Галиуллин 3.Т., Сальников С.Ю., Щуровский В.А. Современные газотранспортные системы и технологии, М.: Газпром ВНИИГАЗ, 2014. [Galiullin Z.T., Salnikov S.Y., Schurovski V.A. Centrifugal compressor machines, Moscow, Gazprom VNIIGAZ, 2014 (in Russian)]

[3] Никитин В.Г., Ишков А.Г., Прокопец А.О., Пошелюзный А.Н., Русинов С.В., Перевозчиков А.Ю., Комаров О.В., Писарев Ю.Н. Повышение эффективности транспорта газа путем примене- 
ния современных высокоэффективных сменных проточных частей центробежных компрессоров. Газовая промышленность спеивыпуск, 2017, 1, 2-9. [Nikitin V.G., Ishkov A.G., Prokopech A.O., Posheluzni A.N., Rusinov S.V., Perevozchikov A.Y., Komarov O.V., Pisarev Y.N. Improving the efficiency of gas transport by means the application of modern high-performance replacement flow parts of centrifugal compressors Gas industry special issue, 2017, 1, 2-9. (in Russian)]

[4] Селезнев К.П., Галеркин Ю.Б. Центробежные компрессоры, Л. Машиностроение, 1982. [Seleznev K.P., Galerkin Y. Centrifugal compressor machines, Leningrad, Machinostroenie, 1982 (in Russian)]

[5] Галеркин Ю.Б. Турбокомпрессоры. СПб., Изд-во КХТ., 2010, 650 с. [Ris V.F. Turbocompressors, SPb, KHT, 2010, 650 p. (in Russian)]

[6] Солдатова К.В. Создание новой математической модели проточной части центробежных компрессоров и базы данных модельных ступеней, дис... д-ра. техн. наук. СПб, 2017, $357 \mathrm{c}$. [Soldatova K. The creation of new mathematical models of the centrifugal compressors flow part and a database of model stages, Dr. of tech. Sci. SPb, 2017, 257 p. (in Russian)]

[7] Дроздов А.А. Метод проектирования центробежных компрессоров с осерадиальными рабочими колесами, дис... канд. техн. наук. СПб, 2016, 236 с. [Drozdov A.A. Design method of centrifugal compressors with 3D impellers, Cand. of tech. Sci. SPb, 2016, 236 p. (in Russian)]

[8] Galerkin Y., Drozdov, A. Centrifugal compressor stage design principlies cheking. ASME 2015 Gas Turbine India Conference, GTINDIA 2015, 2015.

[9] Галеркин Ю.Б., Дроздов А.А. Моделирование газодинамических характеристик центробежных компрессорных ступеней с осерадиальными рабочими колесами. Научнотехнические ведомости СПбГПУ. Наука и образование, 2014, 3(202), 45-53. [Galerkin Y.B., Drozdov A.A. Modeling of gas-dynamic characteristics of centrifugal compressor stages with the 3D impellers, Nauchno-tekhnicheskie Vedomosti SPbGPU. Science and education, 2014, 3(202), 45-53 (in Russian)]

[10] Галеркин Ю.Б., Дроздов А.А. Конструирование и оптимизация центробежной компрессорной ступени с осерадиальным рабочим колесом при помощи инженерного метода проектирования. Научно-технические ведомости СПбГПУ. Наука и образование, 2015, 4(231), 179-188. [Galerkin Y.B., Drozdov A.A. Design and optimization of centrifugal compressor stage with 3D impeller using the engineering design method, Nauchno-tekhnicheskie Vedomosti SPbGPU. Science and education, 2015, 4(231), 179-188 (in Russian)]

[11] Попова Е.Ю. Оптимизация основных параметров ступеней турбомашин на основе математического моделирования. дис... канд. техн. наук. СПб, 1991, 275 с. [Popova Е.Y. Optimization of the main parameters of the turbomachines stages on the basis of mathematical modeling, Cand. of tech. Sci. SPb, 1991, 275 p. (In Russian)]

[12] Денисенко В.В., Писарев Ю.Н., Васильев А.В., Журин А.Г. Опыт разработки, изготовления, доводки и эксплуатации центробежных нагнетателей для ГПА и ЭГПА. ХІІ Международный симпозиум «Потребители-производители компрессоров и компрессорного оборудования - 2006», 2006, 58-60. [Denisenko V.V., Pisarev Y.N., Vasilev A.V., Zhurin A.G. Experience in the development, manufacturing, debugging and operation of centrifugal compressors for GPA and EGPA. XII intermational symposium «Consumers, manufacturers of compressors and compressor equipment - 2006», 2006, 58-60. (in Russian)]

$$
-1077-
$$


[13] Бакаев Б.В., Денисенко В.В., Писарев Ю.Н., Журин А.Г., Левенец С.А., Васильев А.В. Перспективные разработки турбокомпрессорного оборудования в ОАО «Компрессорный комплекс». ХІІІ Международный симпозиум «Потребители-производители компрессоров и компрессорного оборудования - 2007», 2007, 92-96. [Bakaev B.V., Denisenko V.V., Pisarev Y.N., Zhurin A.G., Levenech S.A., Vasilev A.V. The future developments of turbomachinery in "Compressor complex". XIII intermational symposium «Consumers, manufacturers of compressors and compressor equipment - 2007», 2007, 92-96. (in Russian)]

[14] Бакаев Б.В., Денисенко В.В., Писарев Ю.Н., Журин А.Г., Левенец С.А., Васильев А.В. Перспективные разработки турбокомпрессорного оборудования в ОАО «Компрессорный комплекс». XIV Международный симпозиум «Потребители-производители компрессоров и компрессорного оборудования - 2008», 2008, 60-65. [Bakaev B.V., Denisenko V.V., Pisarev Y.N., Zhurin A.G., Levenech S.A., Vasilev A.V. The future developments of turbomachinery in "Compressor complex". XIV intermational symposium "Consumers, manufacturers of compressors and compressor equipment - 2008», 2008, 60-65. (in Russian)]

[15] Бакаев Б.В., Иванов М.Н., Левенец С.А., Васильев А.В., Денисенко В.В., Журин А.Г., Писарев Ю.Н., Хрекин С.Н. Новые разработки турбокомпрессоров для ОАО «Газпром» и других отраслей промышленности. XVI Международный симпозиум «Потребители-производители компрессоров и компрессорного оборудования - 2011», 2011, 30-32. [Bakaev B.V., Иванов М.Н., Levenech S.A., Vasilev A.V., Denisenko V.V., Zhurin A.G., Pisarev Y.N., Chrekin S.N. New development of turbo compressors for "Gazprom" and other industries. XVI intermational symposium «Consumers, manufacturers of compressors and compressor equipment - 2011», 2011, 30-32. (in Russian)] 